\title{
Intrathoracic core needle biopsy and repeat biopsy for PD-L1 evaluation in non-small cell lung cancer
}

\author{
Giulia Maria Stella ${ }^{1}$, Chandra Bortolotto ${ }^{2}$, Andrea Riccardo Filippi ${ }^{3}$ \\ ${ }^{1}$ Department of Medical Sciences and Infectious Diseases, Unit of Respiratory System Diseases, ${ }^{2}$ Department of Intensive Medicine, Unit of Radiology, \\ ${ }^{3}$ Department of Medical Sciences and Infectious Diseases, Unit of Radiation Oncology, Fondazione IRCCS Policlinico San Matteo, Pavia, Italy \\ Correspondence to: Andrea Riccardo Filippi. Department of Medical Sciences and Infectious Diseases, Unit of Radiation Oncology, Fondazione IRCCS \\ Policlinico San Matteo, Pavia, Italy. Email: A.Filippi@smatteo.pv.it. \\ Provenance: This is an invited Editorial commissioned by Executive Editor-in-Chief Jianxing He (Director of the Thoracic Surgery Department, The \\ First Affiliated Hospital of Guangzhou Medical University, Guangzhou, China). \\ Comment on: Tsai EB, Pomykala K, Ruchalski K, et al. Feasibility and Safety of Intrathoracic Biopsy and Repeat Biopsy for Evaluation of Programmed \\ Cell Death Ligand-1 Expression for Immunotherapy in Non-Small Cell Lung Cancer. Radiology 2018;287:326-32.
}

Submitted Sep 05, 2018. Accepted for publication Sep 11, 2018.

doi: $10.21037 /$ jtd.2018.09.92

View this article at: http://dx.doi.org/10.21037/jtd.2018.09.92

Over the past years, the improved knowledge on the biological, genetic and molecular heterogeneity of tumors, together with the development of pharmaceutical technologies, has allowed the identification of several targets for novel therapeutic strategies. This fast process has led to the overall reconsideration of the biological peculiarities that can make each tumor a pathology on its own. Nonsmall cell lung cancer (NSCLC) has been object of extensive research, showing clearly different pathologic and biomolecular features, and an in-depth analysis of tumor genomes and signaling pathways may currently define a set of distinct diseases with specific genetic and cellular features (1). Until recently, genetic mutations or cytogenetic abnormalities (i.e., EGFR mutation, $A L K$ translocation and many other targetable genetic lesions) have been the main drivers for a personalized therapeutic approach (2). As the therapeutic role of immune checkpoint inhibitors targeting programmed death-1 (PD-1 inhibitors, nivolumab, pembrolizumab) and programmed death ligand-1 (PD-L1 inhibitors, atezolizumab, durvalumab) rapidly raised popularity, by showing striking survival gains for NSCLC patients, both when used as single agents or in combination with chemotherapy or chemo-radiotherapy, in second line and first line, PD-L1 expression evaluation and reevaluation on tissue samples became crucial both at diagnosis and after therapy (3). PD-L1 expression is currently assessed by immunohistochemistry (IHC), with a very wide range of positivity, ranging from $1 \%$ to $100 \%$ (4).
While tumor molecular profiling for genetic alterations such as EGFR mutation or $A L K$ translocation became part of the routine diagnostic workflow, we and others started reporting the feasibility and accuracy of tumor mutational screening in aspirated lung cancer cells [fine needle aspiration (FNA)] through imaging guidance (5-7). At the same time many other groups showed the feasibility and reliability of core needle biopsy (CNB) for lung cancer molecular profiling. FNA cytology was recently shown to be as accurate as CNB for PD-L1 testing in retrospective studies $(8,9)$, but a recent study by Tsai and collaborators (10) shed new light on this topic, describing for the first time the feasibility and diagnostic accuracy of CT-guided transthoracic CNB for PD-L1 expression in a prospective series of 110 biopsies derived from the KEYNOTE-001 study population; $91.8 \%$ of these procedures were performed as repeat biopsies subsequent to a previous diagnostic procedure, as pembrolizumab was tested in second line, and $84.5 \%$ were performed after therapy. Results showed that specimens were adequate in $96.4 \%$ of biopsies, and the median number of cores obtained was 8 , consistently higher than previously reported. One of the most interesting findings was that the complication rate was not significantly increased in patients undergoing repeat biopsy after therapy (systemic including pembrolizumab, radiation, surgery), being pneumothorax the most common reported complication.

CNB gained popularity in many centers worldwide 
given the progressive increasing need of tumor material for diagnostic purposes, in parallel to the increasing understanding of the disease biology and the identification of novel actionable targets, with tumor re-biopsies at disease progression being progressively incorporated into clinical practice with the aim to detect acquired resistance to targeted therapies (11). These practice changes have sometimes privileged CT-guided CNB vs. CT-guided FNA. In this scenario, the work by Tsai et al. (10) clearly demonstrates the role of $\mathrm{CNB}$ as an effective method aimed at obtaining tissue for IHC expression analysis of PD-L1, retrieving data from a high-quality prospective patients' series and reporting accuracy and complication rates for mostly second biopsies (91\%), adding important information to the present knowledge. CT-guided FNA might also be ideal for repeated biopsies, but only retrospective data are currently available on its performance in evaluating PD-L1 expression. A theoretical advantage for FNA for repeat biopsies is the expected slightly lower rate of toxicity, however it was generally reported in early uncontrolled retrospective series and a significant difference is not clearly established (12). The two options (CNB and FNA) seems equivalent for molecular subtyping, and studies have shown satisfactory concordance of PD-L1 testing on cell block and cytology smears with histology blocks; however, there are only few reports on performance, utility, and satisfactory results of cytology specimens for PD-L1 testing (13). Two aspects might be underlined in regards to the possible role of FNA: (I) the development of the cell block method, a complementary approach to conventional FNA cytology, has allowed for a higher quality molecular diagnosis $(14,15)$ and may also be used for PD-L1 expression assessment together with direct smear in FNA samples obtained from lung or lymph nodes with transbronchial or transthoracic image guidance, with $2-3$ aspiration specimens (8); (II) the possibility to combine the cell block technique with IHC staining, that has been reported to significantly improve the adequacy of CT-guided FNA samples for standard diagnosis, molecular subtyping and immunosuppressive molecule expression profile, being a convenient strategy in terms of safety and costs (8).

In conclusion, we congratulate Dr. Tsai and collaborators for their precious work, highlighting the current role of CNB for PD-L1 expression evaluation for initial and especially repeat biopsies in advanced stage lung cancer patients who are candidate to immune checkpoint inhibitors, with an acceptable $25 \%$ complication rate. The high number of samples per patient obtained (mean $=8$ ) is safe and seems to be correlated to a high diagnostic accuracy, offsetting lung cancer spatial heterogeneity.

\section{Acknowledgements}

None.

\section{Footnote}

Conflicts of Interest: Dr. C Bortolotto: consulting or advisory role: Bracco Imaging; Dr. AR Filippi: consulting or advisory role: Astra Zeneca; Speakers' Bureau: MSD, Astra Zeneca; Dr. GM Stella has no conflicts of interest to declare.

\section{References}

1. Reck M, Rabe KF. Precision Diagnosis and Treatment for Advanced Non-Small-Cell Lung Cancer. N Engl J Med 2017;377:849-61.

2. Politi K, Herbst RS. Lung cancer in the era of precision medicine. Clin Cancer Res 2015;21:2213-20.

3. McLaughlin J, Han G, Schalper KA, et al. Quantitative Assessment of the Heterogeneity of PD-L1 Expression in Non-Small-Cell Lung Cancer. JAMA Oncol 2016;2:4654. Erratum in: Error in Figure Presentation. [JAMA Oncol 2016].

4. Kerr KM, Hirsch FR. Programmed Death Ligand-1 Immunohistochemistry: Friend or Foe? Arch Pathol Lab Med 2016;140:326-31.

5. Stella GM, Scabini R, Inghilleri S, et al. EGFR and KRAS mutational profiling in fresh non-small cell lung cancer (NSCLC) cells. J Cancer Res Clin Oncol 2013;139:1327-35.

6. Schuurbiers OC, Looijen-Salamon MG, Ligtenberg MJ, et al. A brief retrospective report on the feasibility of epidermal growth factor receptor and KRAS mutation analysis in transesophageal ultrasound- and endobronchial ultrasound-guided fine needle cytological aspirates. J Thorac Oncol 2010;5:1664-7.

7. Ulivi P, Romagnoli M, Chiadini E, et al. Assessment of EGFR and K-ras mutations in fixed and fresh specimens from transesophageal ultrasound-guided fine needle aspiration in non-small cell lung cancer patients. Int J Oncol 2012;41:147-52.

8. Noll B, Wang WL, Gong Y, et al. Programmed death ligand 1 testing in non-small cell lung carcinoma cytology cell block and aspirate smear preparations. Cancer Cytopathol 2018;126:342-52. 
9. Heymann JJ, Bulman WA, Swinarski D, et al. PD-L1 expression in non-small cell lung carcinoma: Comparison among cytology, small biopsy, and surgical resection specimens. Cancer Cytopathol 2017;125:896-907.

10. Tsai EB, Pomykala K, Ruchalski K, et al. Feasibility and Safety of Intrathoracic Biopsy and Repeat Biopsy for Evaluation of Programmed Cell Death Ligand-1 Expression for Immunotherapy in Non-Small Cell Lung Cancer. Radiology 2018;287:326-32.

11. Stella GM, Luisetti M, Pozzi E, et al. Oncogenes in nonsmall-cell lung cancer: emerging connections and novel therapeutic dynamics. Lancet Respir Med 2013;1:251-61.

12. Villar Álvarez F, Muguruza Trueba I, Flandes Aldeyturriaga J. Core Needle Biopsy Versus Fine Needle Aspiration

Cite this article as: Stella GM, Bortolotto C, Filippi AR. Intrathoracic core needle biopsy and repeat biopsy for PDL1 evaluation in non-small cell lung cancer. J Thorac Dis 2018;10(Suppl 33):S4031-S4033. doi: 10.21037/jtd.2018.09.92
Biopsy in Diagnosing Lung Cancer. Arch Bronconeumol 2017;53:655-6.

13. Jain D, Sukumar S, Mohan A, et al. Programmed deathligand 1 immunoexpression in matched biopsy and liquidbased cytology samples of advanced stage non-small cell lung carcinomas. Cytopathology 2018;29:550-7.

14. Kossakowski CA, Morresi-Hauf A, Schnabel PA, et al. Preparation of cell blocks for lung cancer diagnosis and prediction: protocol and experience of a high-volume center. Respiration 2014;87:432-8.

15. Dong Z, Li H, Zhou J, et al. The value of cell block based on fine needle aspiration for lung cancer diagnosis. J Thorac Dis 2017;9:2375-82. 